\title{
Influencia del tipo de anzuelo y la profundidad de pesca en la captura con palangre de tiburones y otras especies pelágicas al noroeste del Pacífico mexicano
}

\author{
Influence of hook type and fishing depth on longline catches of sharks and other pelagic species \\ in the northwest Mexican Pacific \\ Ildefonso Galeana-Villaseñor ${ }^{1}$, Felipe Galván-Magaña² y Roberto Gómez-Aguilar ${ }^{3}$ \\ ${ }^{1}$ Instituto Tecnológico de Mazatlán. Corsario 1 No.203. Col. Urías. Mazatlán, Sinaloa,México. C.P. 82070 \\ ${ }^{2}$ Centro Interdisciplinario de Ciencias Marinas. Av. IPN s/n. Apdo. Postal 592. La Paz, México. C.P. 23000 \\ ${ }^{3}$ Universidad Autónoma de Nayarit. Unidad Académica de Agricultura. Carretera Tepic-Compostela Km 9, Jalisco. Nayarit, \\ México. C.P. 63780 \\ galvan.felipe@gmail.com
}

\begin{abstract}
An experiment using longlines to evaluate the effects of hook type and depth of longline on the catches of sharks and other pelagic species was carried out close to Mazatlan, Mexico. We compared the J-style hooks, tuna hook, tuna $18^{\circ}$ offset hook, and circle hook at three depth strata: $\mathrm{S}_{1}$ (0-55 m), $\mathrm{S}_{2}$ (55-85 m) y $\mathrm{S}_{3}$ (85-99 m). From the total capture, the circle hook had significantly higher catch rate than J-style hook and tuna hook. The main species caught with circle hook were blue marlin (Makaira nigricans) and striped marlin (Tetrapturus audax). The highest biomass of blue shark (Prionace glauca), blue marlin, sailfish (Istiophorus platypterus) and striped marlin was found in the shallow stratum (0-55 m) than in the deep stratum (85-99 m). By hook type, the higher capture of blue marlin was with circle hook and tuna hook at shallow depths $(0-55 \mathrm{~m})$.
\end{abstract}

Key words: Sharks, large pelagics, Gulf of California
Resumen.- Para evaluar el efecto del tipo de anzuelo y la profundidad de colocación del palangre sobre la captura de tiburones y otras especies pelágicas, se realizó un experimento cerca de Mazatlán, México. Se compararon los anzuelos recto plano 8/0, atunero plano 8/0, atunero inclinado 8/0 (18 ${ }^{\circ}$ de inclinación) y circular plano, en tres estratos de profundidad: $\mathrm{S}_{1}(0-55 \mathrm{~m}), \mathrm{S}_{2}$ (55-85 m) y $\mathrm{S}_{3}$ (85-99 m). De la captura total, el anzuelo circular mostró significativamente mayor tasa de captura de marlin azul (Makaira nigricans) y marlin rayado (Tetrapturus audax), comparado con el anzuelo recto y el anzuelo atunero plano. En el estrato de profundidad más somero de los anzuelos (0-55 m) se presentó una mayor tasa de captura y peso de tiburón azul (Prionace glauca), marlin azul, pez vela (Istiophorus platypterus) y marlin rayado comparado con el estrato más profundo (85-99 m). La mayor tasa de captura y peso de marlin azul se presentó con los anzuelos circular y atunero plano en la profundidad más somera del palangre (0$55 \mathrm{~m})$.

Palabras clave: Tiburones, pelágicos mayores, Golfo de California

\section{Introducción}

Los palangres de deriva son ampliamente utilizados en los mares del mundo para la captura de especies pelágicas grandes. En los mares mexicanos se utilizan para la captura de tiburones, pez espada Xiphias gladius y atunes como especies objeto de captura, los cuales incidentalmente capturan tortugas y especies reservadas a la pesca deportiva en México como marlin azul Makaira nigricans, marlin rayado Tetrapturus audax, pez vela Istiophorus platypterus y dorado Coryphaena hippurus. Tradicionalmente se utilizan anzuelos rectos y anzuelos atuneros; sin embargo, existe una tendencia internacional de introducir anzuelos circulares en las pesquerías comerciales para disminuir la mortalidad de especies no objeto de captura, principalmente peces de pico (Istiophoridae y Xiphiidae) y tortugas marinas. Se ha observado que el anzuelo circular retiene con más frecuencia a la presa por la boca, facilitando su liberación y disminuyendo la retención por el tracto digestivo y vísceras, reflejándose en una reducción de la mortalidad (Kerstetter \& Graves 2006). Sin embargo, en la pesquería de palangre en el Océano Pacífico mexicano, los pescadores se oponen al uso del anzuelo circular, por considerar que no tiene la misma efectividad que los anzuelos tradicionales, en la captura de tiburones. Algo similar está sucediendo en un gran porcentaje de las pesquerías pelágicas internacionales de palangre en el Océano Atlántico, donde se continúa el uso del anzuelo recto tradicional (Kerstetter \& Graves 2006). 
La diferencia más obvia entre el anzuelo circular y los convencionales de forma recta, es la orientación de su lanceta, ya que en el anzuelo circular ella está generalmente orientada de forma perpendicular a la caña mientras que en los de forma recta, la lanceta es paralela a la caña. En algunos anzuelos circulares, la punta del anzuelo está dirigida hacia la parte curvada del mismo. Además, el anzuelo circular por tener acortada la caña es más redondeado y de ahí su nombre de circular (Cooke \& Suski 2004).

Con el propósito de inducir el aprovechamiento sostenible de tiburones y rayas, así como de proteger otras especies que son capturadas incidentalmente, el gobierno de México implementó una norma de protección oficial (NOM-029-PESC-2006), que obliga el uso de anzuelos circulares con un tamaño mínimo o superior a $64 \mathrm{~mm}$ de longitud y $22 \mathrm{~mm}$ de abertura (Diario Oficial de la Federación 2007). La FAO ha establecido lineamientos que recomienda que las pesquerías de palangre deben desarrollar e implementar combinaciones de diseños de anzuelos, profundidad de pesca, tipo de carnada y prácticas de pesca que minimicen la captura incidental de tortugas y su mortalidad (FAO 2005).

Se está introduciendo ampliamente el anzuelo circular en las pesquerías comerciales en México; sin embargo, se requiere examinar su efecto en la eficiencia de capturas de las especies como el atún Thunnus spp., pez espada Xiphias gladius y tiburones, así como también evaluar cómo afecta la captura por unidad de esfuerzo (CPUE) de las especies objeto de captura e incidentales (Yokota et al. 2006).

La profundidad en la cual las especies son capturadas es fundamental para entender el impacto en las pesquerías de palangre sobre las especies objeto de captura y la pesca incidental (Bigelow et al. 2006).

Uno de los objetivos principales de la táctica de pesca es colocar el palangre a determinada profundidad de pesca, asociada a la supuesta preferencia de la distribución vertical de la especie objeto de captura (Bach et al. 2006). Además de la profundidad máxima de pesca del palangre, se debe considerar la diferencia de profundidad entre los anzuelos que se distribuyen en cada canasta, debido a que la línea madre toma una forma curvada (línea catenaria). Estas diferencias pueden ocasionar cambios en la composición de la captura (Santana-Hernández et al. 1998).

El presente experimento fue realizado para evaluar el efecto de cuatro tipos de anzuelo, la diferencia de profundidad de pesca de los anzuelos, y la posible interacción anzuelo-profundidad en la pesquería de palangre de deriva para la captura de tiburón en las costas del noroeste del Pacífico mexicano. Se comparó la tasa de captura, peso, porcentaje de máxima evidencia de contacto (\%MC) y el porcentaje de éxito de la máxima evidencia de contacto (\%ECO) de cuatro tipos de anzuelos: recto, atunero plano, atunero inclinado y circular a tres estratos de profundidad de pesca $\mathrm{S}_{1}(0-55$ m), $\mathrm{S}_{2}(55-85 \mathrm{~m})$ y S $_{3}(85-99 \mathrm{~m})$.

\section{Material y métodos}

El experimento se realizó frente a las costas de Mazatlán, México (Fig. 1), del 7 de febrero al 18 de mayo de 2004, en cinco viajes de pesca experimental con un total de 38 lances, a bordo de la embarcación Doña Esthela (14 m eslora total) provista de un tambor hidráulico para almacenar la línea madre del palangre.

El palangre consistió en una línea madre de poliamida (PA) monofilamento de $4 \mathrm{~mm}$ de diámetro con 19,2 km de longitud, con marcas cada $40 \mathrm{~m}$ de distancia (Fig. 2A). Los orinques se construyeron con poliamida monofilamento de $3 \mathrm{~mm}$ de diámetro y una longitud de

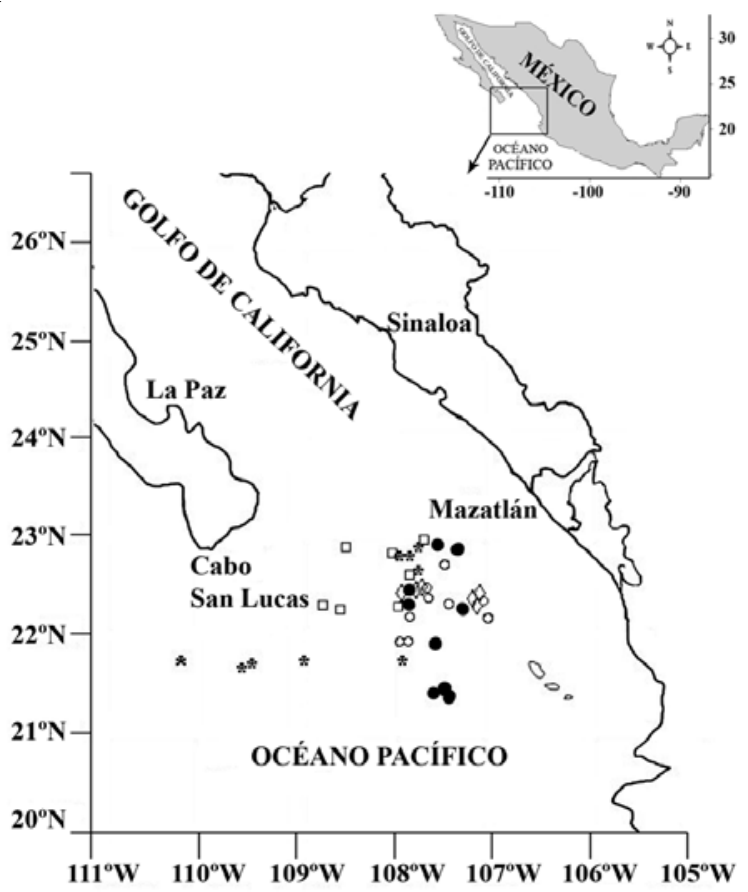

Viajes de pesca experimental $1=*, 2=\square, 3=\diamond, 4=\bullet, 5=0$

Figura 1

Área de estudio y ubicación de 38 lances del palangre de deriva en cinco viajes de pesca experimental

Study area showing the 38 pelagic longline set locations in five experimental fishing trips 


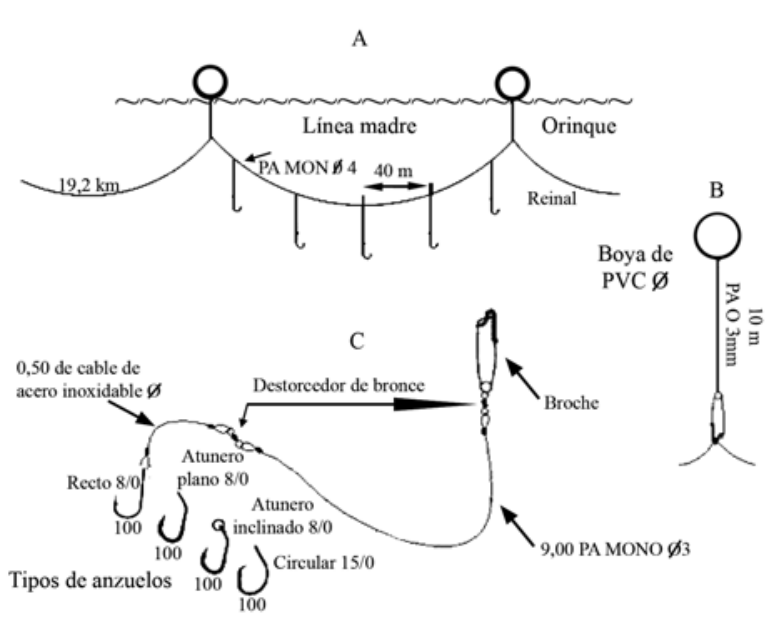

Figura 2

Representación esquemática de: (A) Configuración del palangre, (B) Orinque y (C) Reinal y tipo de anzuelos

Schematic representation of: (A) a section of longline, (B) Buoy line, (C) gangions and hook types

$10 \mathrm{~m}$, con un broche en el extremo para unirse a la línea principal para formar las canastas (Fig. 2B). Los reinales se construyeron en dos secciones, la primera, de poliamida monofilamento de $3 \mathrm{~mm}$ de diámetro con un destorcedor y un broche en el extremo que se unió en cada marca de la línea principal y la segunda, de alambre de acero inoxidable de 1,6 mm de diámetro y 0,50 m de longitud con un destorcedor en un extremo que se unió a la primera sección y en el otro extremo se unió el anzuelo (Fig. 2C). El número de anzuelos en cada canasta (5 anzuelos) se conservó del palangre original que utilizan los pescadores en la zona donde se realizó el experimento (Fig. 2A).

Se utilizaron cuatro tipos de anzuelos: el recto plano $8 / 0$, atunero plano $8 / 0$, atunero $8 / 0$ con $18^{\circ}$ de inclinación (comunes en la pesca comercial) y circular plano 15/0 de una longitud total similar (variación $\pm 5,6 \mathrm{~mm}$ ) y forma diferente (Fig. 3A). Para medir las principales dimensiones del anzuelo se propuso el siguiente criterio: Se tomó la parte recta de la caña del anzuelo como línea de referencia y con líneas paralelas y perpendiculares a esta línea, se midió cada una de las dimensiones (Fig. 3B). El ángulo de ataque se midió considerando la abertura entre la línea de referencia que une la parte central del ojo de la caña y la punta del anzuelo y una línea que es paralela a la superficie de la lanceta del anzuelo. El ángulo de ataque está referido a la posición que el anzuelo toma en el instante en que el pez es enganchado (Baranov 1976) (Fig. 3B). Se consideró el ángulo como positivo (+) si la lanceta del anzuelo tendía a ser paralela a la caña y negativo (-) si tendía a ser perpendicular (Fig. 3B). Las dimensiones que caracterizan la forma de los anzuelos se muestran en la Fig. 3C.

Se utilizó un mismo tipo y tamaño de carnada (juveniles de Mugil cephalus), con una longitud total promedio de 21,8 cm y peso medio de $139,7 \mathrm{~g}$.

El palangre fue calado entre las 6:00 y las 7:00 h cada día dejándolo pescar durante el día para recogerlo entre las 16:00 y 17:00 h.

\section{Profundidad de pesca}

Al colocar el palangre toma una forma curvada en cada canasta, provocando que los anzuelos se encuentren a diferente profundidad (Fig. 2A). Se considera que esta forma curvada es ocasionada por las cargas transversales (peso) que actúan uniformemente distribuidas a lo largo de la línea principal, dando la forma de una línea catenaria (Santana-Hernández et al.1998). La profundidad de pesca del palangre depende de la longitud de la línea por canasta y la combadura de la línea principal entre flotadores que tiende a incrementarse con el HPB (número de anzuelos utilizados por flotador) (Bach et al. 2006). El método para estimar la profundidad de pesca de los anzuelos consistió en determinar la forma de la canasta como una línea catenaria, por medio de la razón de combadura:

$$
\lambda=\frac{l}{\mathbf{L}}
$$

donde $l$ es la distancia entre flotadores de la canasta y $\mathrm{L}$ la longitud de la línea principal (Bucki 1981, Boggs 1992, Bach et al. 2006). La longitud entre flotadores se determinó con $l=\mathrm{t} \bullet \mathrm{v}$, donde $(\mathrm{t})$ es el tiempo en segundos que se midió (antes de iniciar cada cobrado del palangre), en el recorrido de la embarcación entre flotadores de 5 canastas y (v) es su velocidad de desplazamiento. La longitud (L) de la línea principal correspondió a 6 marcas de $40 \mathrm{~m}$ con una longitud total de $240 \mathrm{~m}$. Los valores de $\lambda$ encontrados fluctuaron de 0,510 a 0,773 con un valor medio de 0,675 con el cual se trazó la forma de la canasta utilizando un programa de computadora (CAD) y, con la longitud de los orinques y reinales, gráficamente se determinó la profundidad de pesca de los anzuelos. Las profundidades encontradas fueron de $55 \mathrm{~m}$ para la posición de los anzuelos 1 y 5 del extremo de la canasta, $85 \mathrm{~m}$ para los anzuelos 2 y 4 de la parte media y $99 \mathrm{~m}$ para el anzuelo 3 en la parte central. Con la profundidad media de los anzuelos se establecieron tres estratos de profundad $\mathrm{S}_{1}(0-55 \mathrm{~m}), \mathrm{S}_{2}(55-85 \mathrm{~m})$ y $\mathrm{S}_{3}(85-99 \mathrm{~m}$ ) (Fig. 4). 
Vol. 43, Nº1, 2008

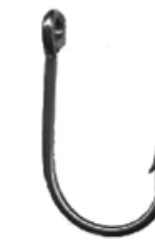

Recto plano $8 / 0$

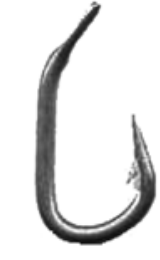

Atunero plano $8 / 0$
A

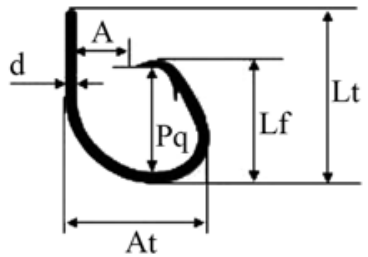

Lt : Longitud total

Lf : Longitud frontal

At : Anchura total

A : Abertura

$\mathrm{Pg}$ : Profundidad de garganta

d : Diámetro de la caña

$\alpha$ : Ángulo de ataque

$\beta$ : Ángulo de inclinación

B
Atunero inclinado $8 / 0$

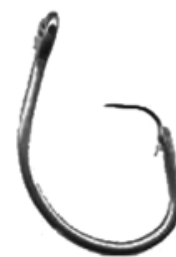

Circular plano $8 / 15$

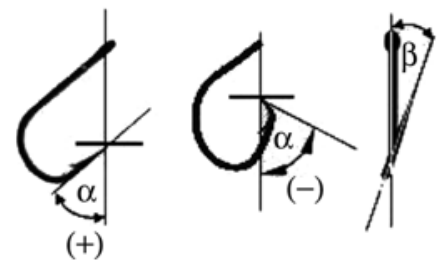

Posición del anzuelo en el instante en que el pez es enganchado (Baranov, 1976)

$\mathrm{C}$

\begin{tabular}{llccccccccccc}
\hline & & \multicolumn{1}{c}{} & \multicolumn{4}{c}{ Dimensión } & mm & \multicolumn{4}{c}{ Ángulo $\left[{ }^{\circ}\right]$} \\
Tipo (nombre común) & Marca Comercial & Tamaño & Lt & Lf & At & A & Pg & d & $\alpha$ & $\beta$ \\
\hline Recto plano & Mustad-95160 & $8 / 0$ & 60,9 & 35,3 & 33,1 & 31,6 & 36,4 & 3 & $54,4(+)$ & 0 \\
Atunero plano & Mustad-9202S & $8 / 0$ & 63,9 & 34,8 & 32,7 & 27,1 & 34,8 & 5,1 & $11,7(+)$ & 0 \\
Atunero inclinado & Mustad-9202SKR & $8 / 0$ & 64,0 & 35,1 & 31,9 & 26,9 & 35,1 & 5,1 & $13,1(+)$ & 17,6 \\
Circular plano & Mustad-3996D & $15 / 0$ & 58,4 & 39,3 & 48,5 & 22,4 & 36,4 & 3,6 & $32,9(+)$ & 0 \\
\hline
\end{tabular}

Figura 3

Características de los anzuelos experimentales. (A) tipo de anzuelos, (B) forma en que se midieron los anzuelos y (C) dimensión de los anzuelos. (+) lanceta del anzuelo paralela a la caña, (-) lanceta perpendicular a la caña

Hook characteristics. (A) Hook types, (B) Hook measures (C) Hook sizes. (+) Spear of the hook parallel to shank, (-) spear of the hook perpendicular to the shank

En el experimento se compararon los cuatro tipos de anzuelo y los tres estratos de profundidad. El palangre se conformó de 80 canastas (400 anzuelos). Los cuatro tipos de anzuelos se distribuyeron al azar en grupo de 100 anzuelos de un mismo tipo (20 canastas) que se ubicaron en los tres estratos de profundidad por cada lance y se realizaron 38 lances o repeticiones.

\section{Registro de datos del experimento}

Se registró la ubicación geográfica del inicio y el final del calado del palangre en cada lance, mediante un Sistema de Posicionamiento Global (GPS).

Durante el cobrado del palangre se registró de manera consecutiva cada reinal que se levantaba, y se recolectaba la siguiente información por tipo de anzuelo: anzuelo con carnada, sin carnada, dañado, perdido ó con captura, anotando la especie y peso. El orden de los anzuelos recolectados permitió ubicarlos posteriormente por su estrato de profundidad de pesca.

Las dos variables que se consideraron para describir la interacción de los organismos con el anzuelo por profundidad de pesca y tipo de anzuelo, se calcularon de la siguiente manera: La primera, tomada del procedimiento descrito por Broadhurst \& Hazin (2001).

$$
\% \mathrm{MC}=\left(\frac{\mathrm{Cf}+\mathrm{Cb}+\mathrm{Cd}}{\mathrm{Cn}}\right) \times 100
$$




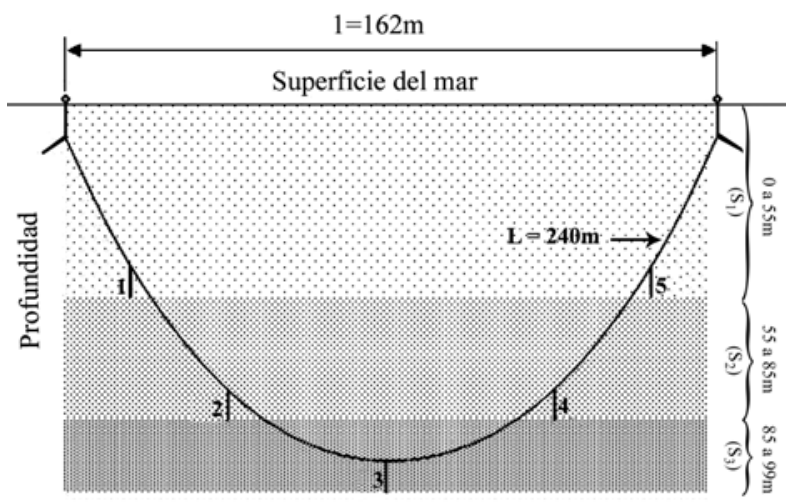

Figura 4

Representación esquemática de la forma estimada que adquiere la canasta del palangre (con la posición de los 5 anzuelos) después de ser calado y los estratos de profundidad medias de pesca de los anzuelos $\left(\mathrm{S}_{1}, \mathrm{~S}_{2}\right.$ y $\left.\mathrm{S}_{3}\right)$ que fueron determinados con la proporción ë=l $/ \mathrm{L}=\mathbf{0 , 6 7 5}$

Schematic representation of the longline basket shape after setting (with five hooks). Hook's fishing depth layer $\left(\mathrm{S}_{1}, \mathrm{~S}_{2} \mathrm{y}\right.$

$\mathrm{S}_{3}$ ) estimated with the ratio sag ë $=\mathrm{l} / \mathrm{L}=0.675$

donde \% MC representa el \% de anzuelos que mostró evidencia de mayor contacto con el pez, Cf es el número de anzuelos que retuvieron el pez, $\mathrm{Cb}$ es el número de anzuelos sin carnada, Cd es el número de anzuelos perdidos y $\mathrm{Cn}$ es el número de anzuelos por tipo y profundidad de pesca.

La segunda variable se propuso como:

$$
\% \mathrm{ECO}=\frac{\mathrm{Cf}}{\mathrm{Cf}+\mathrm{Cb}+\mathrm{Cd}} \times 100
$$

donde \%ECO (éxito de contacto) representa el \% de anzuelos que tuvo éxito en retener la presa del total de anzuelos que mostraron mayor evidencia de contacto (suma de Cf, $\mathrm{Cb}$ y Cd que fueron descritos anteriormente).

\section{Análisis estadístico}

El diseño experimental utilizado fue de bloques al azar; los tratamientos se diseñaron con dos factores considerando el tipo de anzuelo y estrato de profundidad de pesca. El bloque estuvo determinado por el lance. El modelo estadístico fue el siguiente:

$$
y_{i j k}=\mu+S_{i}+H_{j}+D_{k}+(H D)_{j k}+e_{i j k}
$$

donde $y_{i j k}$ es la variable respuesta, ì es la media general, $\mathrm{S}_{i}$ es el lance o repetición, $\mathrm{H}_{j}$ es el efecto del tipo de anzuelo, $\mathrm{D}_{k}$ es el efecto del estrato de profundidad, (HD) ${ }_{j k}$ es el efecto de la interacción entre tipo de anzuelo y estrato de profundidad y $e_{i k j}$ es el error aleatorio.
Para compensar la diferencia en el número de anzuelos del estrato de profundidad 85 a 99 m de cada canasta (un anzuelo), con respecto a los otros estratos de profundidad (dos anzuelos respectivamente), al analizar la información, en lugar de usar las variables número de animales capturados y peso total de la captura, se consideraron la tasa de captura (1) y la tasa del peso total (2), que se calcularon con:

$$
\text { (1) } \mathrm{Tc}=\frac{\mathrm{N}^{\mathrm{o}}}{\mathrm{Nh}} \times 100
$$

donde Tc es la tasa de captura (número de peces/100 anzuelos), $\mathrm{N}^{\circ}$ el número de peces capturados y Nh número de anzuelos utilizados.

$$
\text { (2) } \mathrm{Tp}=\frac{\mathrm{P}}{\mathrm{Nh}} \times 100
$$

donde Tp es la tasa de peso total (kg/100 anzuelos), P es el peso total de la captura en $\mathrm{kg}$ y $\mathrm{Nh}$ el número de anzuelos utilizados.

Como las variables \%MC, \%ECO y Tc son valores discretos, fueron transformadas con $\operatorname{Ln}(\mathrm{x}+1)$ para inducir normalidad (Berry 1987, Yokota et al. 2006).

Para el análisis estadístico, el valor de la variable \%ECO, en los lances donde todos los anzuelos salieron con carnada y sin captura, se consideró como celda vacía o dato perdido. Se utilizó el Modelo Lineal Generalizado (GLM) para el análisis de los datos y la prueba de Tukey para las comparaciones múltiples de medias. Asimismo, se utilizó la prueba de Duncan para los resultados de ANOVA que mostraron diferencias significativas, pero que con la prueba de Tukey no presentó diferencias en las medias.

\section{Resultados}

Durante el experimento se capturó un total de 589 organismos con un peso total de 17273,2 kg (Tabla 1). El tiburón azul (Prionace glauca) fue la especie dominante representando el $58,6 \%$ de la captura total. El resto de la captura estuvo compuesta por un pequeño número de otros tiburones $(6,4 \%)$, peces de pico $(23,6 \%)$, dorado $(8,1 \%)$, rayas $(1,9 \%)$, atún $(1,0 \%)$ y tortugas $(0,05 \%)$.

En la Tabla 2 se presenta un resumen de los resultados del ANOVA para los dos factores y las diferentes variables de estudio. Sólo se muestran los resultados de las especies que presentaron diferencias significativas en alguna de las variables de tasa de captura y peso. Los datos de las otras especies que se analizaron y no presentaron diferencias significativas fueron Coryphaena hippurus 


\section{Tabla 1}

Número y peso total de especies capturadas durante el experimento

Total number and weight of species caught during the experiment

\begin{tabular}{lcr}
\hline Especie & Número & Peso (kg) \\
\hline Tiburón azul (Prionace glauca) & 345 & 10374,5 \\
Marlin azul (Makaira nigricans) & 80 & 2657,5 \\
Dorados (Coryphaena hippurus) & 48 & 437,0 \\
Marlin rayado (Tetrapturus audax) & 31 & 1356,4 \\
Tiburón zorro (Alopias pelagicus) & 25 & 1049,5 \\
Pez vela (Istiophorus platypterus) & 24 & 694,0 \\
Rayas (Dasyatis violacea) & 11 & 67,0 \\
Tiburón mako (Isurus oxyrinchus) & 10 & 192,0 \\
Atún aleta amarilla (Thunnus albacares) & 6 & 51,0 \\
Pez espada (Xiphias gladius) & 4 & 212,0 \\
Cornuda prieta (Sphyrna zygaena) & 2 & 42,0 \\
Tortuga prieta (Chelonia agassizi) & 2 & 45,3 \\
Tiburón prieto (Carcharhinus obscurus) & 1 & 95,0 \\
Total & 589 & 17273,2 \\
\hline
\end{tabular}

y Alopias pelagicus. Por el escaso número de captura, no fue posible examinar los datos de otras especies como los tiburones Isurus oxyrinchus, Sphyrna zygaena y Carcharhinus obscurus; la raya Dasyatis violacea; la tortuga marina Chelonia agassizi; el pez espada Xiphias gladius y el atún Thunnus albacares.

\section{Comparación del tipo de anzuelo}

La comparación de los cuatro tipos de anzuelos muestra que existen diferencias significativas $(P<0,05)$ en \%MC (Tabla 2). Al aplicar la prueba de Tukey se encontraron diferencias entre los anzuelos circular y atunero inclinado, siendo superior la media del anzuelo circular en 38,9\% (Fig. 5A). Los tipos de anzuelos no presentaron diferencias significativas en \%ECO $(P>0,05)$ (Tabla 2$)$; sin embargo, el porcentaje promedio de anzuelos que tuvo mayor éxito en retener la presa fue el anzuelo circular; mientras que el menor porcentaje fue para el anzuelo atunero plano en un 47\% (Fig. 5B). De las capturas totales, el tipo de anzuelo que presentó diferencias altamente significativas en la tasa de captura $(P<0,01)$ fue el anzuelo circular (media de 2,19 peces /100 anzuelos), no encontrándose diferencias entre los anzuelos recto, atunero plano y atunero inclinado (Fig. 5C). Asimismo no se encontró diferencia en la tasa del peso total según el tipo de anzuelo (Tabla 2).

En el análisis por especie, los anzuelos presentaron diferencias significativas en la tasa de captura de marlin azul $(P<0,05)$, marlin rayado $(P<0,01)$ y peso de marlin rayado $(P<0.05)$ (Tabla 2$)$. En la prueba de Tukey, el anzuelo circular presentó una media superior tanto en la tasa de captura de marlin azul (165,2\% que el anzuelo recto) y marlin rayado (140,5\% que el anzuelo atunero plano) (Fig. 5D), como en el peso de marlin rayado (388,2\% que el anzuelo atunero plano) (Fig. 5E).

Comparación de los tres estratos de profundidad de pesca de los anzuelos $S_{1}\left(0-55 \mathrm{~m}, \mathrm{~S}_{2}(55-85 \mathrm{~m})\right.$ y $\mathrm{S}_{3}(85-$ $99 \mathrm{~m})$

Aplicando la prueba de ANOVA se registraron diferencias significativas entre los estratos de profundidad de los anzuelos para \% MC $(P<0,01)$; \% ECO $(P<0,05)$; tasa de captura total $(P<0,01)$, tasa de captura de tiburón azul $(P<0,05)$, marlin azul $(P<0.01)$ y pez vela $(P<0,05)$; peso de tiburón azul $(P<0,001)$, marlin azul $(P<0,01)$, marlin rayado $(P<0,05)$ y pez vela $(P<0,01)$ (Tabla 2$)$.

Tabla 2

Resumen de los valores $P>F$ de la prueba de ANOVA para determinar el efecto en las variables debido a los lances, diferentes tipo de anzuelos (recto, atunero plano, atunero inclinado y circular) y estratos de profundidad de pesca (0-55, 55-85 y 85-99 m)

Summary of $P>F$ from ANOVA test to determine effects on variables due to set, hook-types, (J-style hook, tuna hook, tuna $18^{\circ}$ offset hook, circle hook) and hook’s fishing depth layers (0-55, 55-85 y 85-99 m)

\begin{tabular}{|c|c|c|c|c|c|c|c|c|c|c|c|c|c|}
\hline \multirow[t]{2}{*}{ Factor } & \multirow[t]{2}{*}{ d.f. } & \multirow[t]{2}{*}{$\% \mathrm{MC}^{\mathrm{a}}$} & \multirow[t]{2}{*}{$\% \mathrm{ECO}^{\mathrm{a}}$} & \multicolumn{2}{|c|}{ Captura total } & \multicolumn{2}{|c|}{ Tiburón azul } & \multicolumn{2}{|c|}{ Marlin azul } & \multicolumn{2}{|c|}{ Marlin rayado } & \multicolumn{2}{|c|}{ Pez vela } \\
\hline & & & & $\mathrm{Tc}^{\mathrm{a}}$ & $\mathrm{Tp}$ & $\mathrm{Tc}^{\mathrm{a}}$ & $\mathrm{Pe}$ & $\mathrm{Tc}^{\mathrm{a}}$ & $\mathrm{Pe}$ & $\mathrm{Tc}^{\mathrm{a}}$ & $\mathrm{Pe}$ & $\mathrm{Tc}^{\mathrm{a}}$ & $\mathrm{Pe}$ \\
\hline Lance & 37 & 0,0001 & 0,0001 & 0,0001 & 0,0001 & 0,0001 & 0,0001 & 0,0001 & 0,0001 & 0,0001 & 0,0001 & 0,0001 & 0,0076 \\
\hline Anzuelo & 3 & 0,0347 & 0,1707 & 0,0053 & 0,0773 & 0,9409 & 0,6868 & 0,0296 & 0,1251 & 0,0093 & 0,0101 & 0,2240 & 0,2779 \\
\hline Estrato & 2 & 0,0001 & 0,0168 & 0,0001 & 0,0638 & 0,0139 & 0,0001 & 0,0093 & 0,0066 & 0,2323 & 0,0472 & 0,0223 & 0,0084 \\
\hline Anz*Est & 6 & 0,3481 & 0,9188 & 0,5209 & 0,1831 & 0,7419 & 0,2933 & 0,0236 & 0,0008 & 0,3469 & 0,2983 & 0,6207 & 0,6977 \\
\hline Error & 222 & & & & & & & & & & & & \\
\hline
\end{tabular}



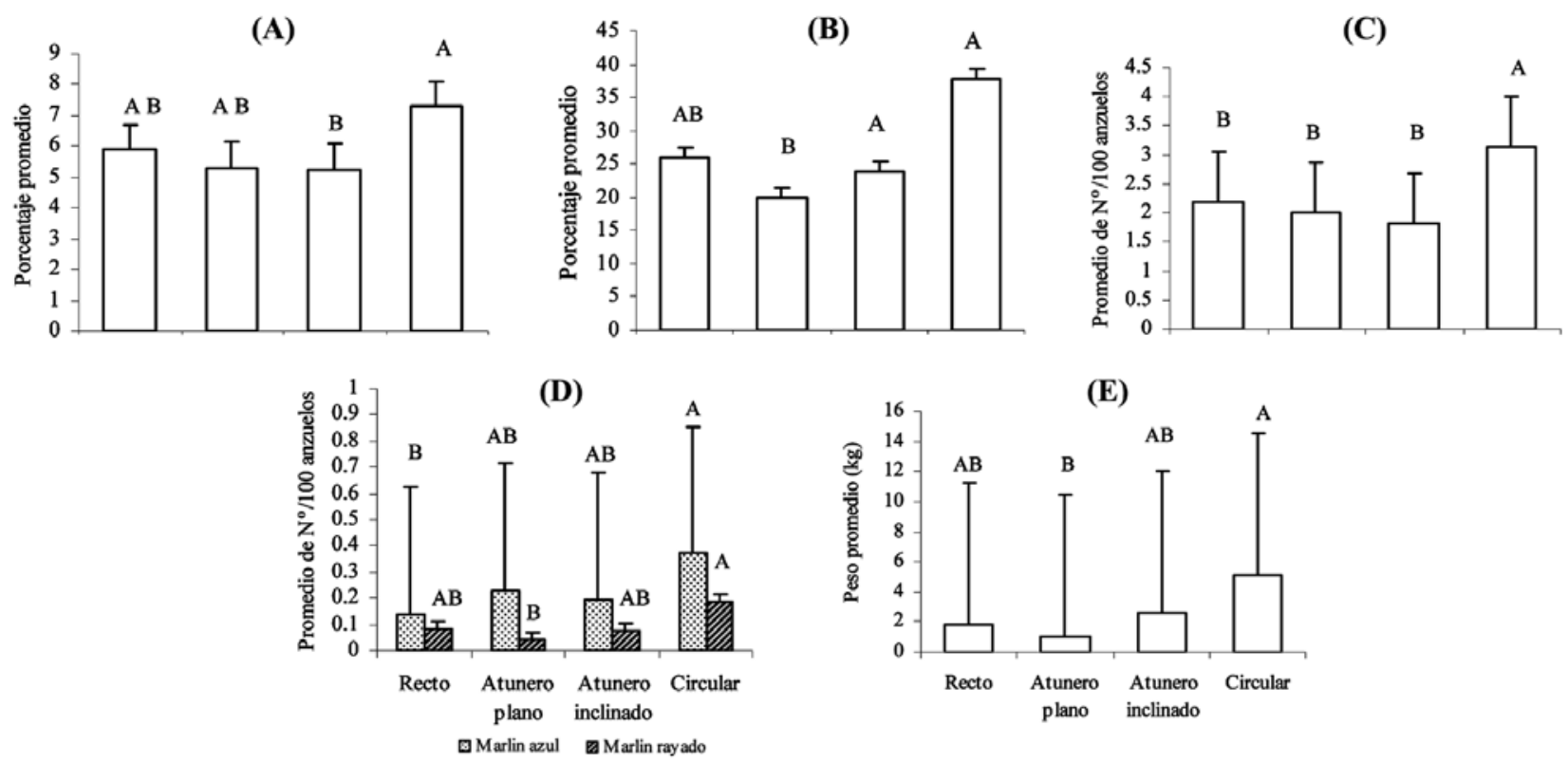

Figura 5

Diferencias en los valores medios ( \pm E.S.) de cuatro tipos de anzuelos para: A) \% de de mayor evidencia de contacto (\%MC), B) \% de éxito de la mayor evidencia de contacto (\%ECO), C) tasa de captura total, (D) tasa de captura de marlin azul y marlin rayado, E) peso de marlin rayado. En la comparación de medias, letras iguales no son significativamente diferentes (por especies, la comparación por anzuelo es para una misma especie)

Differences in mean values $( \pm$ S.E.) between four hook types for: (A) \% of hooks with evidence of major contact (\%MC), (B) \% of hooks that caught the fish out of the total number of hooks that showed evidence of major contact by fish (\%ECO), (C) total catch rate, (D) catch rate of blue marlin and striped marlin, (E) weight of striped marlin. Means with the same letter are not significantly different. (Comparison by hook is within species)

De la captura total, en las comparaciones múltiples (prueba de Tukey), el primer estrato de profundidad $\mathrm{S}_{1}$ presentó medias superiores para \%MC (siendo diferentes

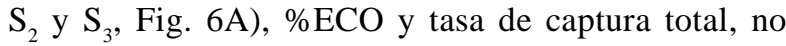
resultando diferentes los estratos $\mathrm{S}_{2} \mathrm{y} \mathrm{S}_{3}$ para estas dos últimas variables (Fig. 6B y C).

En la comparación por especie, los estrato $\mathrm{S}_{1}$ y $\mathrm{S}_{3}$ resultaron diferentes para la tasa de captura de tiburón azul, marlin azul y pez vela, siendo superior la media para el estrato $S_{1}$ en 56,9\% para tiburón azul, 139,9\% para marlin azul y 236,1\% para pez vela, no encontrándose diferencias entre los estratos $\mathrm{S}_{1}$ y $\mathrm{S}_{2}$ (Fig. 6D). Los estratos $S_{1}$ y $S_{3}$ resultaron diferentes para el peso de tiburón azul y peso de marlin azul, siendo superior para el estrato $S_{1}$ las medias del peso del tiburón azul en un $75,9 \%$ y $160,7 \%$ para marlin azul, no encontrándose diferencias del estrato $S_{2}$ con $S_{1}$ y $S_{3}$ (Fig. 6E). El estrato $\mathrm{S}_{1}$ resultó superior a los estratos $\mathrm{S}_{2}$ y $\mathrm{S}_{3}$ (no siendo diferentes entre sí) para el peso de marlin rayado (prueba de Duncan) y pez vela (Fig. 6E).

\section{Interacción entre los anzuelos y los estratos de profundidad}

El ANOVA mostró que existe interacción entre los tipos de anzuelos y sus estratos de profundidad de pesca en la tasa de captura $(P<0,05)$ y peso $(P<0,001)$ de marlin azul (Tabla 2). El anzuelo atunero plano presentó una marcada tendencia de disminución del valor medio de la tasa de captura y peso de marlin azul al aumentar su profundidad de pesca, siendo la disminución de la tasa de captura y peso de $69,9 \%$ y $69,2 \%$ respectivamente al pasar de $S_{1}$ a $\mathrm{S}_{2}$, no registrándose captura en el estrato $\mathrm{S}_{3}$. El anzuelo circular presentó una tendencia similar al pasar del estrato $\mathrm{S}_{1}(0-55 \mathrm{~m})$ al estrato $\mathrm{S}_{2}(55-85 \mathrm{~m})$ disminuyendo tanto la tasa de captura como el peso de marlin azul (62,2\% y $70,7 \%$ respectivamente), pero al aumentar la profundidad de pesca en el estrato $\mathrm{S}_{3}(85-99 \mathrm{~m})$ aumentó la tasa de captura y peso de marlin $(58,9 \%$ y $78,2 \%$ respectivamente); sin embargo, la tasa de captura y peso del marlin azul del estrato $\mathrm{S}_{3}$ resultó inferior al del estrato $\mathrm{S}_{1}$ en un $60,1 \%$ (Fig. $7 \mathrm{~A}$ y $\mathrm{B}$ ). El anzuelo atunero 
(A)

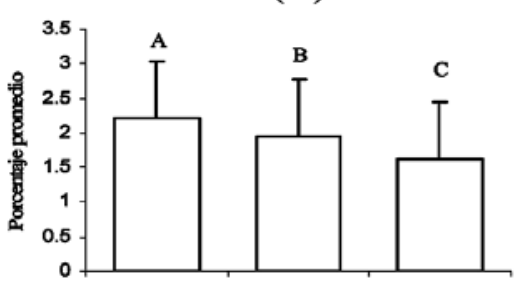

(B)

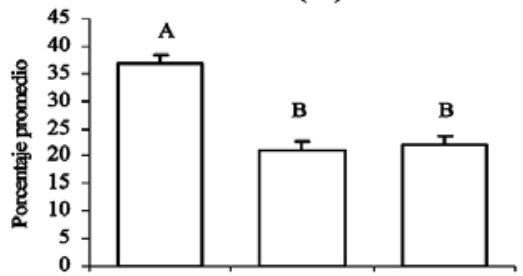

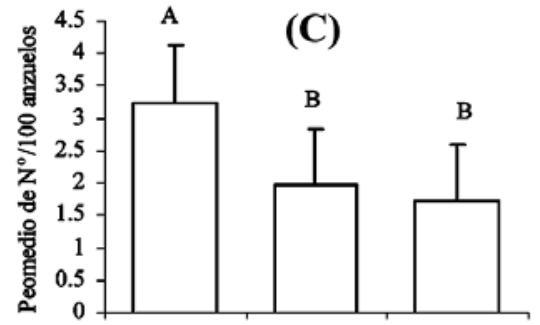

(E)
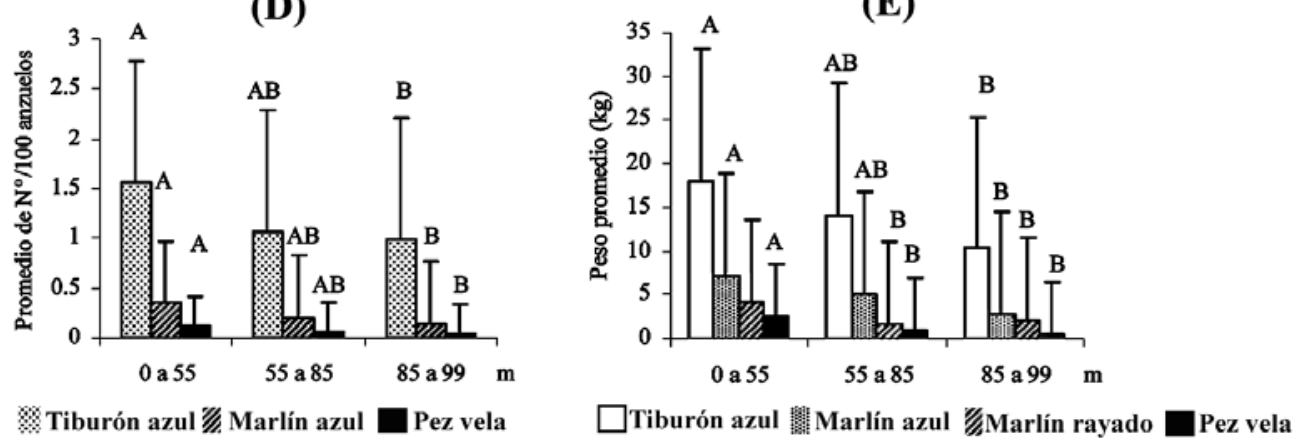

Figura 6

Diferencias en los valores medios $( \pm$ E.S.) de los estrato de profundidad de los anzuelos para: (A) \% de máxima evidencia de captura, B) \% de éxito de máxima evidencia de contacto, (C) tasa de la captura total, D) tasa de captura de tiburón azul marlin azul y pez vela $F$ ) peso de tiburón azul, marlin azul, marlin rayado y pez vela. $S_{1}\left(0\right.$ a 55 m), $S_{2}$ (55 a 85 m) y $S_{3}(8$ a 99 m). En la comparación de medias, letras iguales no son significativamente diferentes (por especies, la comparación por estratos es para una misma especie). *Prueba de Duncan

Differences in mean value ( \pm S.E.) between hooks fishing depth layer of: (A) \% of hook with major contact, B) \% of hooks that caught fish out of the total number of hooks that showed evidence of major contact by fish, (C) total catch rate, D) catch rate of blue shark, blue marlin and sailfish, F) weight of blue shark, blue marlin, striped marlin an sailfish. $\mathrm{S}_{1}(0$ a $55 \mathrm{~m}), \mathrm{S}_{2}(55$ a $85 \mathrm{~m})$ y $\mathrm{S}_{3}(8 \mathrm{a} 99 \mathrm{~m})$. Means with the same later are not significantly different (Comparison by layer is for a same species). *Duncan test

inclinado, por el contrario, presentó un aumento de la tasa de captura y peso de marlin azul al pasar de $\mathrm{S}_{1}$ a $\mathrm{S}_{2}$ (41,8\% y 93,9\% respectivamente) y una disminución de la tasa de captura y peso de marlin azul al pasar de S2 a S3 (58,3\% y 80,2\% respectivamente). El anzuelo recto en comparación con los otros tipos de anzuelos presentó poca variación en la tasa de captura de marlin azul por el efecto de la profundidad de pesca (Fig. 7A), presentando un ligero incremento al pasar del estrato $\mathrm{S}_{1}$ al $\mathrm{S}_{3}(16,4 \%)$; mientras que el peso promedio de marlin azul aumentó de $S_{1}$ a $S_{2}(163 \%)$ pero disminuyendo $(17,2 \%)$ en el estrato $\mathrm{S}_{3}$ (Fig. 7B). El mayor valor medio de la tasa de captura y peso de marlin azul se presentó en el estrato de profundidad de 0-55 m, con los anzuelos atunero plano y circular (Fig. 7A). Por el contrario, la menor tasa de captura y peso de marlin se presentó en el estrato de profundidad de 85-99 m, con el anzuelo atunero plano (Fig. 7B).

\section{Discusión}

De la composición de la captura obtenida con el palangre de deriva, el tiburón azul fue la especie dominante con un 58,6\% de la captura total. Carrera-Fernández (2004) menciona que el tiburón azul representa el 95\% de la captura total en Baja California Sur, México. De acuerdo con las conclusiones del Plan de Acción Nacional para el Manejo y Conservación de Tiburones, Rayas y Especies Afines en México, se indica que el tiburón azul tiene potencial de captura para la flota de mediana altura y de altura (CONAPESCA-INP 2004).

\section{Tipo de anzuelos}

Una de las características más importante de la forma de los anzuelos para la captura es su ángulo de ataque, el cual es la inclinación de la posición que el anzuelo presenta en el instante en que el pez es capturado (Fig. 3B). De acuerdo con la teoría de diseño, Baranov (1976) 

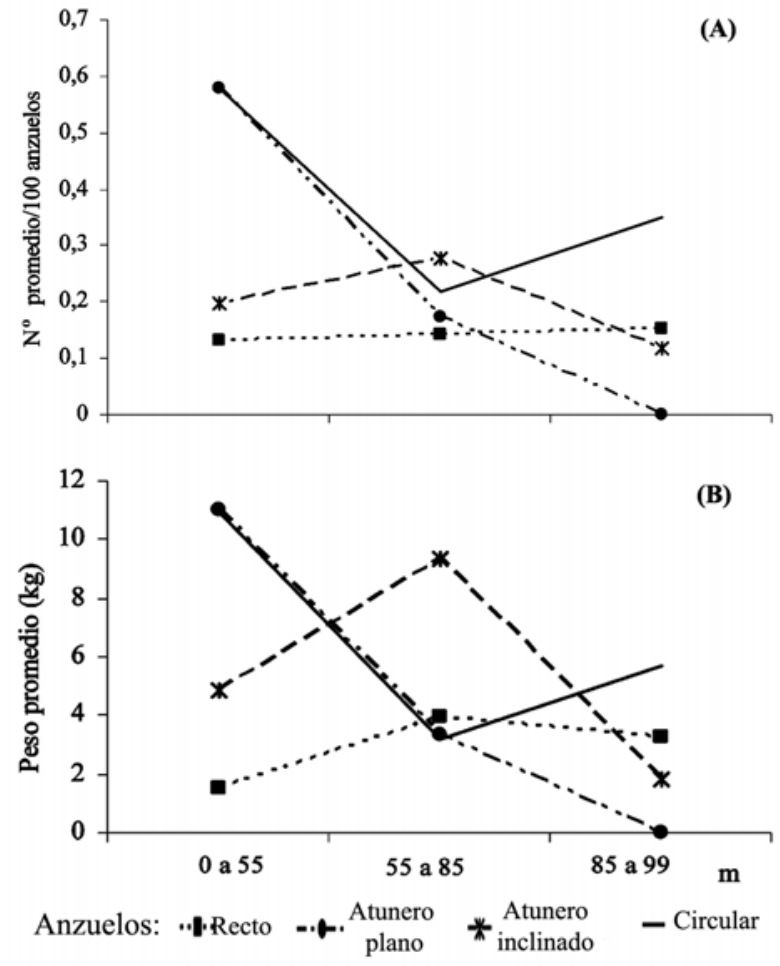

Figura 7

Interacción entre los tipos de anzuelos (recto, atunero plano atunero inclinado y circular) y los estratos de profundidad de pesca S1 (0-55 m), S2 (55-85 m) y S3 (85-99 m) en: A) tasa de captura de marlin azul y $B$ ) peso de marlin azul

Interaction between hook types (J-style hooks, Tuna hook, tuna $18^{\circ}$ offset hook, circle hook) and depth layer S1 (0-55 m), S2 (55-85 m) and S3 (85-99 m). A) Blue marlin catch rate, and B) Blue marlin weight

considera que el éxito del anzuelo para enganchar la presa depende de su ángulo de ataque. Los anzuelos sometidos a experimentación presentaron diferencias en el ángulo de ataque (Fig. 3C); sin embargo, no se observaron diferencias en el \%MC y de tasa de captura. Aunque se debe considerar que en la prueba de Tukey, el anzuelo circular presentó una media superior de \%ECO que el anzuelo atunero plano (Fig. 5B), lo que no concuerda con la teoría del diseño, ya que el anzuelo atunero plano tiene un menor ángulo de ataque $\left(11,7^{\circ}\right)$, en comparación con anzuelo circular $\left(-57,1^{\circ}\right)$. Probablemente el ángulo de ataque tenga influencia en el instante de enganche; sin embargo, la retención de las especies capturadas puede estar influenciada por otros factores como la reacción del pez al anzuelo encarnado (LØkkeborg 1994, Kaimmer 1999), la ubicación de enganche del anzuelo en las especies capturadas (Cooke \& Suski 2004, Kerstetter \& Graves 2006) y el diseño del anzuelo, que por sus características mecánicas, en el instante en que penetra el tejido de la presa, tienda a generar un mayor ángulo de giro en relación a la dirección del jalón, dificultando su liberación (Johannes 1981).

Los resultados del experimento indican que el anzuelo atunero plano en comparación con el anzuelo circular, presentó un porcentaje mayor $(21,7 \%)$ de anzuelos dañados y sin carnada. El anzuelo circular, por su forma redondeada, con la lanceta dirigida perpendicular hacia la caña, proporciona un ángulo de ataque negativo (Fig. 3B), lo cual permite un mayor ángulo de giro cuando la presa está enganchada y trata de escapar, dificultando su liberación. Sin embargo, es difícil determinar cuál forma del anzuelo es mejor, debido a las condiciones de su operación (Baranov 1976).

En el análisis por especie, los resultados de la experimentación muestran que los tipos de anzuelos recto, atunero plano, atunero inclinado y circular no presentaron influencia en la tasa de captura y peso del tiburón azul y tiburón zorro (Alopias pelagicus). No fue posible examinar los datos de otros tiburones como Isurus oxyrinchus, Sphyrna zygaena y Carcharhinus obscurus debido al insuficiente numero de organismos capturados. El reducido impacto que presentó el tipo de anzuelo en la tasa de captura del tiburón azul concuerda con los resultados obtenidos por Yokota et al. (2006) en un estudio realizado en Japón, utilizando un anzuelo atunero convencional (tamaño estándar japonés; 3,8 sun) y dos anzuelos circulares (4,3 sun y 5,2 sun), el cual no encontró diferencia significativa en la tasa de captura de tiburón azul; sin embargo, la tasa de captura fue afectada significativamente por las diferencias en la operación. Pocos experimentos han investigado el efecto del anzuelo circular en la tasa de captura de tiburones pelágicos (Yokota et al. 2006). Sin embargo, en pesquerías donde el tiburón no es la especie objeto de captura, como en las pesquerías comerciales de líneas con anzuelo para serránidos de Carolina del Norte, Estados Unidos de América, Bacheler \& Bukel (2004) evaluaron cuatro tipos y tamaños de anzuelos (rectos de tamaño 5/0, 7/0, 9/0 y circular 12/0), y encontraron variaciones en la captura diaria (ANOVA; $F=3.67$, d.f. $=17, P<0,01$ ) y por el tipo de anzuelo (ANOVA; $\mathrm{F}=3.01$, d.f. $=3, P<0,038$ ) de la captura de tiburones.

El impacto principal del tipo de anzuelo se presentó para las especies no objeto de captura, encontrándose una diferencia significativa en la tasa de captura de marlin azul $(P=0,296)$ y marlin rayado $(P=0,093)$ y peso de marlin rayado $(P=0,101)$. El anzuelo circular resultó con una media superior tanto en la tasa de captura de marlin azul (165\% mayor que el anzuelo recto), marlin rayado 
(140,5\% mayor que el anzuelo atunero plano) como en el peso del marlin rayado (388,5\% mayor que el anzuelo atunero plano). Mientras que en la captura de dorado (Coryphaena hippurus) se tuvo un número significativo de captura; sin embargo, el tipo de anzuelo no tuvo influencia en su tasa de captura y peso. De acuerdo con los resultados obtenidos, es claro que la implementación del anzuelo circular en la pesquería de palangre de deriva para la captura de tiburones no afecta su tasa de captura y peso; sin embargo, con el interés de reducir la captura incidental de peces de pico se debe considerar estos resultados, ya que el anzuelo circular tiene una fuerte influencia en el incremento de la tasa de captura de marlin azul y marlin rayado, así como en peso de marlin rayado, en comparación con los anzuelos recto y atunero plano. Adicionalmente al tipo de anzuelo, la zona de pesca tiene importancia en la pesca incidental de peces de pico ya que su abundancia relativa en determinadas áreas se puede reflejar en la tasa de captura (Goodyear 1990). La razón principal por la que se está implementando ampliamente el uso del anzuelo circular es para reducir la mortalidad de la pesca incidental (Cooke \& Suski 2004, Kerstetter \& Graves 2006). El reciente interés en el anzuelo circular ha sido beneficioso para estimular el interés y la investigación en el papel que juega el diseño del anzuelo en la reducción del daño y la mortalidad causada por el enganche del anzuelo en la liberación de las especies capturadas (Cooke \& Suski 2004).

\section{Estratos de profundidad de pesca}

El conocimiento de la distribución horizontal y vertical de las especies pelágicas es un dato importante para establecer las estrategias de pesca con el palangre de deriva. La distribución vertical de los peces en la columna de agua está relacionada principalmente con la intensidad de la luz, la presión, temperatura y la concentración de oxígeno (Fréon \& Misund 1999). Una adecuada superposición entre la distribución vertical de la especie objeto de captura y la distribución de la profundidad de pesca de los anzuelos puede aumentar la probabilidad de captura (Bach et al. 2006). El conocimiento de la profundidad de nado de grandes peces pelágicos es importante para la evaluación de la CPUE en las pesquerías de palangres.

Considerando los resultados de la captura total, la diferencia en la profundidad de pesca de los anzuelos presentó diferencia significativa en el porcentaje de evidencia de mayor contacto del pez (\%MC, $P=0,0001$ ), porcentaje de captura del \%MC (\%ECO, $P=0,0168)$ y tasa de captura $(P=0,0001)$. Los valores medios tanto del \%MC, \%ECO y de la tasa de captura, fueron superiores para la profundidad más somera $\left(\mathrm{S}_{1}=0-55 \mathrm{~m}\right)$ y sólo para el \%MC los estratos de profundidad $\mathrm{S}_{2}$ (55$85 \mathrm{~m})$ y S $_{3}(85-99 \mathrm{~m})$ fueron diferentes. Estos resultados muestran evidencia de que la mayoría de las especies capturadas se encontró distribuida en la profundidad de pesca más somera de los anzuelos del palangre (0-55 m), cuando menos durante el tiempo de fijado del palangre. Es probable que este comportamiento de captura corresponda a los hábitos alimentarios de los depredadores al preferir las capas superficiales para buscar el alimento. También se debe considerar que algunas especies como el dorado y peces de pico son capturados durante el calado y cobrado del palangre (Boggs 1992).

Las tasas de captura de tiburón azul, marlin azul y pez vela presentaron diferencias significativas en relación a la profundidad de pesca de los anzuelos, siendo su valor medio superior en el estrato de profundidad de 0-55 m (Fig. 6d). Se debe considerar que el experimento se realizó con pesca diurna entre las 6:00 y las 17:00 h. Se han realizado estudios para determinar la distribución vertical del marlin azul por medio de un sensor. Block \& Booth (1992) encontraron que durante el día el marlin azul se mantuvo cerca de la superficie a una temperatura de $27^{\circ} \mathrm{C}$ nadando a velocidades menores a $50 \mathrm{~cm} \mathrm{~s}^{-1}$; mientras que durante la noche se sumerge a mayores profundidad entre 50 y $100 \mathrm{~m}$. Sin embargo, para el marlin rayado, Brill et al. (1993) encontraron, por medio de telemetría ultrasónica, que no presenta diferencias en su distribución vertical entre el día y la noche, lo cual también concuerda con los resultados obtenidos en este trabajo, al no encontrar diferencias significativas de su tasa de captura en los tres estratos de profundidad de los anzuelos (Tabla 2).

La distribución de las capturas obtenidas con el experimento deben tomarse con precaución debido a que los estratos de profundidad de los anzuelos en el palangre fueron determinados con los valores medios de la razón de combadura (ë) y no se verificaron con dispositivos de registro de temperatura-profundidad (TDRs), como se han hecho con otros experimentos similares (Boggs 1992, Santana-Hernández et al. 1993, Bigelow et al. 2006), ya que la profundidad del palangre es afectada por el viento y las corrientes oceánicas que pueden hundirlo o levantarlo (Bigelow et al. 1999), pudiendo modificarse durante el experimento la profundidad de pesca estimada.

\section{Interacción entre los anzuelos y los estratos de profundidad}

Se han realizado estudios para determinar la distribución vertical de las especies pelágicas (Block \& Booth 1992, Brill et al. 1993), la profundidad de pesca del palangre 
de deriva (Trujillo et al. 2003, Bach et al. 2006, Bigelow et al. 2006), la relación de la profundidad de pesca del palangre con los resultados de captura (Boggs 1992, Santana-Hernández 1998) y el efecto que el tamaño y tipo de anzuelo tiene en los resultados de captura (Kerstetter \& Graves 2006, Yokota et al. 2006). Sin embargo, también tiene importancia en los resultados de captura la influencia del tipo de anzuelo asociado con su profundidad de inmersión.

En el experimento no se presentó interacción anzueloprofundidad $(P>0,05)$ en la captura total (en el \%MC, \%ECO y Tc); sin embargo, en la tasa de captura y peso de especies de tiburón, marlin rayado, pez vela y marlin azul sí se vieron afectadas por la combinación anzueloprofundidad $(P<0,05)$. Los resultados mostraron que la mayor tasa de captura se obtuvo con los anzuelos circular y atunero plano en la profundidad más somera del palangre (0-55 m), disminuyendo para el siguiente estrato de profundidad (55-85 m). El anzuelo circular aumentó la tasa de captura en el estrato (85-99 m) mientras que el anzuelo atunero plano en ese estrato no registró capturas (Fig. 7A). De acuerdo a la posición del anzuelo en la canasta del palangre, las mayores tasas de captura y peso del marlin azul se obtuvieron con los anzuelos circular y atunero plano ubicados en los reinales más cercanos a los orinques; mientras que la menor tasa de captura y peso del marlin azul fue con el anzuelo atunero plano ubicado en la parte media de la canasta (Fig. 4). La norma mexicana NOM-029-PESC-2006 para la protección de tiburones y rayas contempla el uso obligatorio de anzuelos circulares con tamaño 15/0 ó 16/0 por lo menos en los reinales más cercanos a los orinques (Diario Oficial de la Federación 2007). De acuerdo con los resultados obtenidos, el uso de anzuelos circulares en los reinales próximos a los orinques como lo establece la norma mexicana, incrementaría la tasa de captura incidental de marlin azul y marlin rayado en comparación con el uso de anzuelo recto y atunero inclinado. Si los resultados obtenidos son confirmados por más estudios, se podría considerar que el uso de anzuelo recto en sus diferentes estratos de profundidad podría reducir la tasa de captura incidental de marlin azul con la posibilidad de que no se presenten diferencias significativas en la tasa de captura de tiburón azul.

\section{Agradecimientos}

Al Cosnet, Instituto Tecnológico de Mazatlán y al Instituto Politécnico Nacional (COFAA y EDI) por su apoyo en la realización del proyecto. A los ingenieros Samuel Garay Ortiz y Abel René Pérez Mejía por su participación en la pesca experimental, así como a dos evaluadores anónimos.

\section{Literatura citada}

Bach P, P Travassos \& D Gaertner. 2006. Why the number of hooks per basket (HPB) is not a good Proxy indicator of the maximum fishing depth in drifting longline fisheries? Collective Volume of Scientific Papers ICCAT 59(2): 701715.

Bacheler NM \& JA Buckel. 2004. Does hook type influence the catch rate, size, and injury of grouper in a North Carolina commercial fishery? Fisheries Research 69: 303311.

Baranov FI. 1976. Selected works on fishing wear. Volume I. Commercial fishing techniques, $631 \mathrm{pp}$. Israel Program for Scientific Translation, Jerusalem.

Berry DA. 1987. Logarithmic transformations in ANOVA. Biometric 43: 439-456.

Bigelow KA, CH Bogas \& X He. 1999. Environmental effects on swordfish and blue shark catch rate in the US North Pacific longline fishery. Fisheries Oceanography 8(3): 178198.

Block BA \& D Booth. 1992. Direct measurement of swimming speed and depth of blue marlin. Journal of Experimental Biology 166: 267-284.

Boggs CH. 1992. Depth, capture time, and hooked longevity of longline-caught pelagic fish: Timing byte of fish with chips. Fishery Bulletin 90: 642-658.

Brill RW, DB Holts, RKC Chang, S Sullivan, H Dewar \& FG Carey. 1993. Vertical and horizontal movements of striped marlin (Tetrapturus audax) near the Hawaiian Island, determined by ultrasonic telemetry, with simultaneous measurement of oceanic currents. Marine Biology 117: 567-574.

Broadhurst MK \& FH Hazin. 2001. Influences of type and orientation of byte on catch of swordfish (Xiphias gladius) and other species in an artisanal sub-surface longline fishery off northeastern Brazil. Fisheries Research 53: 169-179.

Bucki F. 1981. Principios Generales de Cálculo de las Artes de Pesca. Serie de Materiales Didácticos en Ciencia y Tecnología del Mar, 77 pp. Secretaría de Educación Pública, México.

Carrera-Fernández M. 2004. Biología reproductiva del tiburón azul Prionace glauca (Linnaeus, 1758 en la costa occidental de Baja California Sur, México. Tesis de Maestría en Ciencias, CICIMAR-IPN, México, 67 pp.

CONAPESCA-INP. 2004. Plan de Acción Nacional para el Manejo y Conservación de Tiburones, Rayas y Especies Afines en México, 80 pp. Comisión Nacional de Acuacultura y Pesca e Instituto Nacional de la Pesca, Secretaría de Agricultura, Ganadería, Desarrollo Rural, Pesca y Alimentación. Mazatlán, México. 
Cooke SJ \& CD Suski. 2004. Are circle hooks an effective tool for conserving marine and freshwater recreational catch-and-release fisheries? Aquatic Conservation: Marine and Freshwater Ecosystems 14: 299-326.

Diario Oficial de la Federación. 2007. Norma oficial mexicana. NOM-029-PESC-2006. Pesca responsable de tiburones y rayas. Especificación para su aprovechamiento, 43 pp. Miércoles 14 de febrero de 2007. Primera sección. Secretaria de Gobernación, Mexico.

Food and Agriculture Organization of the United Nations (FAO). 2005. Report of the Technical Consultation on Sea Turtle Conservation and Fisheries. FAO Fisheries Report 765: 1-31.

Fridman AL. 1976. Theory and design of commercial fishing gear. 489 pp. Israel Program for Scientific Translation, Jerusalem.

Goodyear CP. 1999. An analysis of possible utility of timearea closures to minimize billfish bycatch by U.S. pelagic longline. Fishery Bulletin 97: 243-255.

Johannes RE. 1981. Words of the Lagoon: Fishing and Marine Lore in the Palau District of Micronesia, 245 pp. University of California Press, Los Angeles.

Kaimmer S. 1999. Direct observation on the hooking behavior on Pacific halibut, Hippoglossus stenolepis. Fishery Bulletin 97: 873-883.
Kerstetter DW \& JE Graves. 2006. Effects of the circle versus J-style hooks on target and no-target species in a pelagic longline fisheries. Fisheries Research 80: 239-250.

Kooke SJ \& CD Suski. 2004. Are circle hooks an effective tool for conserving marine and freshwater recreational catch-and-release fisheries. Case studies and reviews. Aquatic Conservation: Marine and Freshwater Ecosystems 14: 299-326.

LØkkeborg S. 1994. Fish behavior and longline. En: Fernö A \& S Olsen (eds). Marine fish behavior in capture an abundance estimation, pp. 9-27. Fishing New Books, Oxford.

Santana-Hernández H, R Macías-Zamora \& JJ ValdezFlores. 1998. Selectivity of the longline system used by the Mexican fleet in the Exclusive Economic Zone. Ciencias Marinas 24(2): 193-210.

Trujillo E \& G Martínez. 2003. Estimación de la profundidad de trabajo de anzuelos de un palangre atunero prototipo de la flota atunera de Isla de Margarita (Venezuela). Investigaciones Marinas 31(2): 25-34.

Yokota K, M Kiyota \& H Minami. 2006. Shark catch in a pelagic longline fishery: comparison of circle and tuna hooks. Fisheries Research 81: 337-341.

Recibido el 29 de octubre de 2007 y aceptado el 23 de enero de 2008 